\section{Diffuse pulmonary fibrosis and the Hermansky-Pudlak syndrome: clinical course and postmortem findings}

\author{
S P Reynolds, B H Davies, A R Gibbs
}

\begin{abstract}
The Hermansky-Pudlak syndrome consists of albinism, platelet function defect, pigment laden macrophases and, on occasions, pulmonary fibrosis. The clinical course and postmortem findings of a patient with pulmonary fibrosis which mimicked cryptogenic fibrosing alveolitis are reported. Histological examination revealed a chronic inflammatory infiltrate of pigment laden microphages.
\end{abstract}

(Thorax 1994;49:617-618)

The triad of albinism, platelet function defect, and pigment laden macrophages is known as the Hermansky-Pudlak syndrome. ${ }^{1}$ The occurrence of pulmonary fibrosis as part of this syndrome has been emphasised by Davies and Tuddenham. ${ }^{2}$

We report the clinical case and postmortem findings of a 51 year old woman with oculocutaneous albinism, a platelet function defect, and slowly progressive diffuse interstitial pulmonary fibrosis who was previously reported in 1976 as the index case of a family study. ${ }^{2}$

\section{Case report}

A woman, aged 35 years at presentation, attended in 1974 with oculocutaneous albinism and a one year history of gradually increasing dyspnoea. There was a three year history of menorrhagia and lifelong easy bruising. Of the nine siblings three others were albinos, two of whom had platelet release defects and diffuse interstitial pulmonary fibrosis. There were no risk factors for allergic alveolitis. She smoked 10 cigarettes daily and was on no medication. She was, on examination, an albino, with white body hair, unpigmented skin, and had nystagmus. She was clubbed and had widespread inspiratory crackles.

Routine haematological biochemical tests and erythrocyte sedimentation rate were normal. No autoantibody or precipitins to alveolitic antigens were detected. Coagulation studies showed a classical platelet release defect: a prolonged bleeding time, increased prothrombin consumption, and absent secondary wave of platelet aggregation with adrenaline.

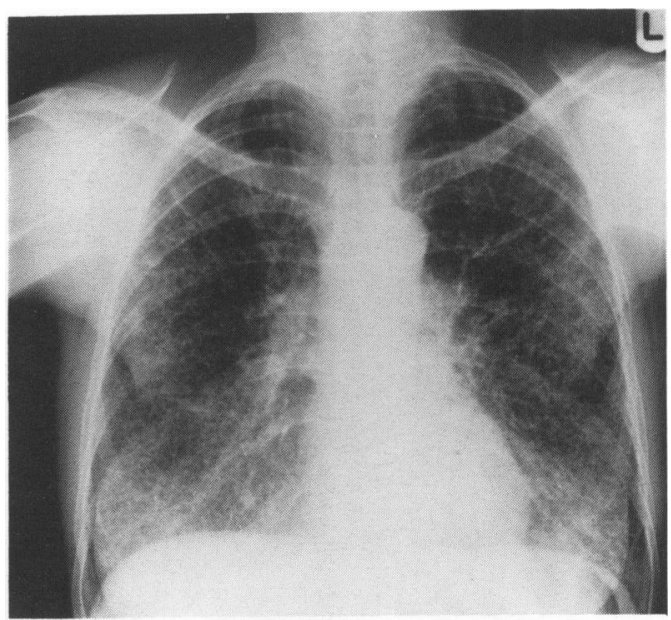

Figure 1 Chest radiograph showing diffuse interstitial fibrosis with honeycombing particularly affecting the upper zones.

Aggregation in response to collagen was much reduced implying a defective platelet release deficit. A bone marrow aspirate confirmed the presence of macrophages with coarse pigment granules staining positive for ceroid. Chest radiography showed diffuse interstitial fibrosis (fig 1) and lung function tests confirmed decreased gas transfer ( $48 \%$ of predicted) and normal static lung volumes. Prednisolone was given at a high initial dose without significant improvement in lung function over six months.

Over the next eight years she progressively deteriorated with increased dyspnoea and decline in lung volumes and gas transfer so that by 1982 her transfer factor was only $27 \%$ of normal and chest radiography showed honeycomb lung. This slow deterioration continued until she died 16 years after presentation.

Postmortem examination showed small lungs with bossellation of the pleural surfaces. There was widespread honeycombing of equal severity throughout the lungs, the cystic spaces appearing larger in the upper lobes. Microscopic examination of the lung showed widespread interstitial fibrosis with disorganised architecture, bronchiolectasis, and loss of alveoli. In the intersitium there was a chronic inflammatory infiltrate with large numbers of pigment laden macrophages (fig 2). The pigment showed similar histochemical properties to lipofuscin. These macrophages and others with staining for iron were present in the alveolar spaces. Organising thrombi were present in the small pulmonary arteries. Similar macrophages were found in other organs including the bone marrow, spleen, liver, kidney, brain, and renal tubules.

\section{Discussion}

The association of oculocutaneus albinism and platelet release deficit, inherited as an autosomal recessive trait, was made in 1959 by Hermansky and Pudlak, ${ }^{1}$ who subsequently reported diffuse lung fibrosis in one of their patients. ${ }^{3}$ It has since been recognised in patients from Wales, ${ }^{2}$ Holland, ${ }^{4}$ Puerto Rico, ${ }^{5}$ and Scandinavia. ${ }^{6}$ We have identified 18 other

\footnotetext{
Asthma and Allergy Penarth, South S P Reynold
Received 24 August 1992 Returned to author 22 October 1992
Revised version received 14 December 1992 Accepted for publication 3 March 1994
Eisease Research Group and A R Gibb
Reprint requests to: Pathology, Llandough
} 


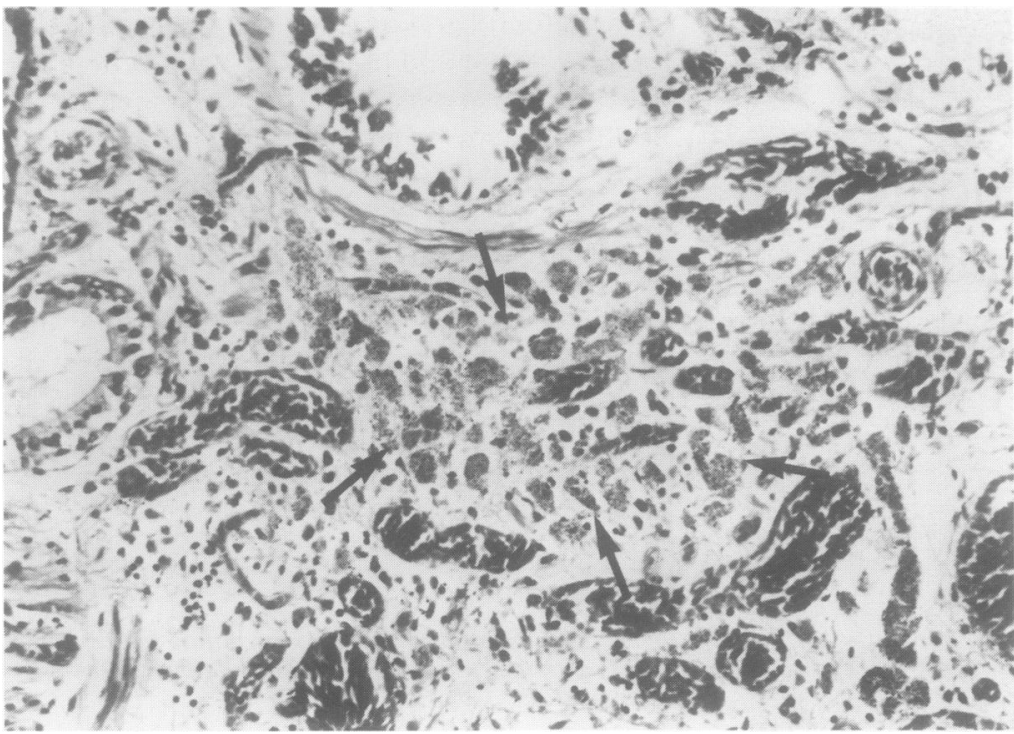

Figure 2 Photomicrograph of interstitium showing chronic inflammatory infiltrate with large numbers of pigment laden macrophages. Original magnification $\times 250$, reduced to $63 \%$ in origination.

cases with associated pulmonary fibrosis, ${ }^{12-10}$ only two of which underwent postmortem examinations. ${ }^{18}$ The pulmonary disease appears to be twice as common in women, ${ }^{10}$ occurring between the third and fourth decades. ${ }^{5}$ Onset of dyspnoea may range from weeks to years and there may be associated weight loss..$^{5}$ Progression to end stage fibrosis and death is recognised. ${ }^{10}$ Chest radiography reveals ill defined densities giving a ground glass appearance progressing to a diffuse reticulonodular pattern and honeycombing. ${ }^{2510}$ Pulmonary function is similar to that of other causes of parenchymal lung disorders.

The pathogenesis of the syndrome is un- clear. The hallmark of the syndrome is ceroid lipofuscin like inclusions throughout the entire reticuloendothelial system. Ceroid, a complex chromolipid, originates from oxidation of unsaturated fatty acids, accumulation resulting from possibly excessive phagocytosis of unsaturated lipids and/or congenital inadequacy to catabolise these lipids. ${ }^{5}$ Although pulmonary macrophages exhibit ceroid deposition, it is unclear whether ceroid is a passive byproduct or induces fibrosis. Alternatively, recurrent haemorrhage with resulting haemosiderosis has been suggested as a possible mechanism for pulmonary fibrosis. ${ }^{2}$

1 Hermansky F, Pudlak P. Albinism associated with haemorrhagic diathesis and unusual pigmented reticular cells in the bone marrow: report of two cases with histochemical studies. Blood 1959;14:162-9.

2 Davies BH, Tuddenham EGD. Familial pulmonary fibrosis associated with oculocutaneous albinism and platelet function defect. A new syndrome. $Q \mathcal{F} M$ ed 1976;178:21932 .

3 Bednar B, Hermansky F, Lojda Z. Vascular pseudohemophilia associated with ceroid pigmentoplagia in albinos. Am ₹ Pathol 1964;45:283-91.

4 Witkop Jr CJ, White JG, King RA. Oculocutaneous albinism. In: Nyham WL, ed. Heritable disorders of amino acid metabolism. New York: John Wiley, 1974:177

5 Garay SM, Gardella JE, Fazzini EP, Goldring RM. Hermansky Pudlak syndrome. Pulmonary manifestations of a ceroid storage disorder. Am $f$ Med 1979;66:737-47.

6 Hoste P, Williams J, Devriendt J, Lamont H, Van Der Straeten M. Familial diffuse interstitial pulmonary fibrosis associated with oculocutaneous albinism. Scand $f$ Respir Dis 1979;60:126-34

7 White DA, Walker Smith EJ, Cooper Jr JD, Glickstein M Rankin JA. Hermansky Pudlak syndrome and interstitia lung disease: report of a case with lavage findings. $A m R e v$ Respir Dis 1984;130:138-41.

8 Takahashi A, Yokoyama T. Hermansky Pudlak syndrome with special reference to lyosomal dysfunction. A case report and review of the literature. Virchows Arch A report and review of the literature. Virchor
Pathol Anat Histopathol 1984;402:247-58.

9 Schinella RA, Greco MA, Cobert BL, Denmark LW, Cox RP. Hermansky Pudlak syndrome with granulomatous colitis. Ann Intern Med 1980; 92: 20-3.

10 Depinho RA, Kaplan KL. The Hermansky Pudlak syndrome. Report of three cases and review of pathophysiology and management considerations. Medicine (Baltimore) 1985;64:192-202.

\section{Osteochondroma of the}

Royal Brompton

National Heart and Lung Hospital, London SW3 6HP

N K Harrison

J Wilkinson

D Hansell

M N Sheppard

P G Goldstraw

A J Newman Taylor

Southend Hospital, Westcliff-on-Sea,

Essex

J O'Donohue

A G Davison

Reprint requests to:

Dr N K Harrison.

Received 20 April 1993

Returned to authors

28 May 1993

Revised version received

5 August 1993

5 August 1993

17 August 1993

\section{rib: an unusual cause of haemothorax}

N K Harrison, J Wilkinson,

J O'Donohue, D Hansell,

M N Sheppard, P G Goldstraw,

A G Davison, A J Newman Taylor

\author{
Abstract \\ The case is described of a 36 year old \\ woman who presented with a large left \\ sided haemothorax. A thoracic computed \\ tomographic (CT) scan suggested there \\ was a bony outgrowth arising from the
}

fourth rib. This was resected surgically and found to be an osteochondroma which was surrounded by blood clot. No definite site of bleeding was identified, but it is thought that the tumour may have traumatised the lung, the pericardiacophrenic artery, or the superior pulmonary vein, resulting in life threatening haemorrhage.

(Thorax 1994;49:618-619)

Primary tumours of the thoracic cage are rare, accounting for only $7-8 \%$ of primary bone tumours. ${ }^{12}$ In a recent series of 90 primary bone tumours affecting the thorax four were osteochondromas, but only one of these occurred in a rib. ${ }^{3}$ We report an osteochondroma of the fourth rib which presented as a large haemothorax. 